\title{
De l'héparine et des inventions de la médecine en général
}

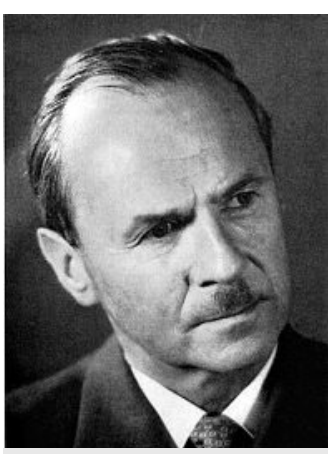

Karl Lenggenhager (1903-1989) défend l'origine suisse de la prophylaxie héparinique.

Un grand merci à mon collègue Thomas Schlich (Université McGill, Montréal).

* La bibliographie peut être consultée dans la version en ligne sur le site www.saez.ch $\rightarrow$ Numéro actuel ou $\rightarrow$ Archives $\rightarrow 2011 \rightarrow 28 / 29$.

** PD Dr ès sc. soc Eberhard Wolff est licencié en sciences culturelles, historien de la médecine et membre de la rédaction Histoire de la médecine du Bulletin des médecins suisses.
Un léger faux pas dans le noir, il y a quelques années, a eu pour moi des effets inattendus. Si la fracture de l'os métatarsien et le plâtre qu'on m'a posé aux urgences étaient en soi des expériences assez inédites, celle de devoir «me piquer» pendant quelques jours l'était au moins autant: je découvrais la thromboprophylaxie sous-cutanée.

«Quoi de plus normal?», se dira le praticien. Posttraumatique ou postopératoire, la thromboprophylaxie est devenue une évidence, explique Urs Lenggenhager, chirurgien à Berne. L'omettre équivaudrait à une faute professionnelle. Ce qu'on sait moins, ajoute-t-il, c'est que la prophylaxie héparinique a de solides origines helvétiques: son père Karl Lenggenhager (1903-1989), médecin à l'hôpital de l'Ile à Berne, s'est consacré dès le début des années 30 à percer les mystères des plaquettes et de la coagulation, un domaine dans lequel il a publié de nombreux travaux. Devenu médecin-chef à l'hôpital de l'Ile, il y a introduit en 1941 le traitement héparinique de 5 injections consécutives dans les 72 heures comme procédure générale de thromboprophylaxie postopératoire, avec des résultats remarquables. C'était il y a 70 ans, et il n'est pas vain de rappeler aujourd'hui ce fait historique à la rédaction du BMS.

«Qui l'a inventé?» - un Suisse, comme dans la publicité pour Ricola? Une brève recherche en ligne sur l'introduction de l'héparine donne des résultats très disparates. Une encyclopédie suisse attribue à Lenggenhager l'introduction de la prophylaxie par héparine faiblement dosée [1]. En 1996, le Dr Felix Mahler, angiologue à Berne à l'époque, écrit que les bases expérimentales de cette pratique ont «probablement» été définies par Lenggenhager et mentionne parallèlement l'utilisation d'héparine par un chirurgien de Stockholm, Clarence Crafoord [2]. Une source allemande relève la simultanéité des premières applications cliniques par les deux chirurgiens en 1935 [3]. Deux auteurs suédois, quant à eux, mettent toute l'emphase sur les études cliniques de Crafoord, tout en évoquant deux autres études pionnières parallèles menées à Toronto [4], tandis qu'une source canadienne - eh oui! - ne mentionne que ces dernières, réalisées par le chirurgien Gordon Murray [5, 6]. En 1960 déjà, l'hebdomadaire hambourgeois «Der Spiegel» révélait à ses lecteurs quel hôpital avait été le «premier du monde» à utiliser régulièrement l'héparine à partir de 1948. On s'en doutait: c'était la clinique gynécologique universitaire de Hambourg [7].

Etablir les origines d'une invention est souvent une affaire de perspective. L'histoire de la médecine nous en donne un autre exemple, plus notoire. Une recherche sur Google de la séquence «fondateur de la bactériologie» en français donne presque deux fois plus de résultats associés au nom de «Pasteur» qu'à celui de «Koch». Si par contre, on saisit la séquence en allemand «Begründer der Bakteriologie» dans le masque de recherche, ce rapport est inversé et atteint franchement deux pour un.

«Qui l'a inventé?» La question se pose de façon moins aiguë pour l'histoire de la médecine qu'elle s'était posée aux stratèges publicitaires de Ricola, parce que le développement en médecine est généralement fait de processus complexes qu'on ne peut réduire à des «Eurêka!» ou à des «Je me lance!» d'individus isolés. Mais c'est justement ce qui rend l'exercice si passionnant! Du reste, la prophylaxie héparinique n'est pas non plus tombée du ciel pour les premiers opérateurs. Il a d'abord fallu isoler chimiquement l'héparine (c'était chose faite en 1916), puis découvrir son effet anticoagulant. Il a ensuite fallu trouver un procédé pour la produire en grande quantité sous forme pure, non toxique, et à un prix abordable [8]. Même une première utilisation en clinique du traitement héparinique n'impliquait aucunement son acceptation par la communauté médicale, ni a fortiori son intégration dans la pratique quotidienne. Celle-ci n'est intervenue qu'au terme d'études à grande échelle dans les années 70 [3].

Le fils de Lenggenhager lui-même observe que cette implémentation a été longue, bien que son père (et les autres collègues travaillant sur le sujet, E. W.) aient publié leurs résultats à maintes reprises. Le fils l'explique en invoquant que son père «n'était pas homme à se faire une publicité tapageuse». Mahler pense qu'une des raisons se trouve dans le fait que les travaux étaient publiés en langue allemande ou qu'en 1941 (!), l'organe de publication Helvetica Medica Acta paraissait chez un éditeur allemand (Thieme, Leipzig) [3].

Les questions de ce genre sont du pain béni pour les Science Studies qui tentent systématiquement de s'aider de leur répertoire méthodique pour rendre plausibles des réponses relevant au départ de la spéculation. Le Bernois Karl Lenggenhager a certainement compté parmi les pionniers de l'introduction de la prophylaxie héparinique. Sa contribution à la médecine bernoise pourrait être vue comme un paradigme de la complexité des inventions dans l'histoire de la médecine.

Eberhard Wolff **

P.S.: cela vaut d'ailleurs aussi pour les bonbons aux herbes! 


\section{Références}

1 www.hls.ch

2 Mahler F. Aus der Geschichte der Thromboseverhütung und -behandlung. Therapeutische Umschau. 1996;53:254-7.

3 Kujath P. Die ambulante Thromboseprophylaxe Deutsches Aerzteblatt. 1995;92:Aa2003-6. www. aerzteblatt.de/pdf/92/28/a2003.pdf

4 Escobar Kvitting JP, Olin CL. Clarence Crafoord. A Giant in Cardiothoracic Surgery, the First to Repair Aortic Coarctation. Ann Thorac Surg. 2009;87:342-6. http://ats.ctsnetjournals.org/cgi/content/ full/ $/ 87 / 1 / 342$
5 Rutty C. Miracle Blood Lubricant. Connaught and the Story of Heparin, 1928-1937. Contact. 1996;9. www.healthheritageresearch.com/HeparinConntact9608.html

6 Marcum JA. The Development of Heparin in Toronto. J Hist Med Allied Sci. 1997;52:310-37. Marcum mentionne également au passage les études de Stockholm, mais pas celles de Berne.

7 Dünnes Blut. Der Spiegel. 27.7.1960. www.spiegel.de/ spiegel/print/d-43067605.html

8 Marcum JA. The Origin of the Dispute over the Discovery of Heparin. J Hist Med Allied Sci. 2000;55:3766. http://muse.jhu.edu/journals/jhm/summary/ v055/55.1marcum.html 